\title{
Biografias de homens e mulheres autores de violência: uma revisão bibliográfica sobre o uso de métodos com trajetória para o entendimento do fenômeno sociológico
}

\author{
Biographies of men and women perpetrators of violence: a \\ bibliographical review about the use of trajectory methods to \\ understand the sociological phenomenon
}

\begin{abstract}
Resumo: Neste artigo, está o resultado de uma revisão da literatura sobre trajetórias de homens e mulheres que cometeram crime ou violência. O objetivo foi analisar o trabalho empírico publicado nos últimos dez anos com uso de biografias para o entendimento do fenômeno social. Os bancos de dados utilizados para a pesquisa foram o Scopus e o SciELO. O corpus final de análise é uma apanhado de 56 documentos. Entre os resultados está a presença de vitimização na trajetória biográfica como um fator que contribui para o cometimento de crimes posteriores por muIheres e homens. Da mesma forma, a socialização do gênero - como um processo vinculado às formas identitárias e à atribuição de significados - aparece na literatura como um aspecto central para a compreensão desse fenômeno. Isso foi identificado na pesquisa qualitativa que utilizou biografias.
\end{abstract}

Palavras-chave: Violência; Crime; Homens; Mulheres; Biografia.

\begin{abstract}
This paper presents the results of a literature review about trajectories of men and women who have committed crimes or violent actions. The objective is to analyse empirical research published in the last ten years that employed the biographical approach to understand this social phenomena. Literature was gathered using Scopus and SciELO electronic libraries. The final corpus included 56 documents. In the analysis of biographical trajectories, victimisation appears to be a contributing factor in the perpetration of crimes by both men and women. Furthermore, gendered socialisation - seen as a process linked to identity and assignment of meanings - appears in literature as a key aspect in the understanding of this phenomenon.
\end{abstract}

Keywords: Violence; Crime; Men; Women; Biography.

\section{Introdução}

Os estudos sobre violência e criminalidade têm uma longa trajetória de pesquisa na área das Ciências Sociais. Diferentes abordagens e metodologias são usadas para trabalhar essa temática 
(BRETAS, 1991). Nos últimos anos, o interesse pelo assunto cresceu, o que fez ampliar também o conhecimento a respeito dos fenômenos (TAVARES DOS SANTOS, 2007). Os sociólogos vêm se perguntando os fatores que fazem com que os sujeitos escolham mais recorrentemente usar a violência para resolver conflitos, frustrações, falta de algo material ou simbólico (GROSSI; AZEVEDO, 2013).

A violência não é necessariamente um crime em todas as legislações e períodos históricos se consideramos que não é apenas física, mas resultado de uma relação de poder em que um impõe sua força em detrimento de outro, seja de maneira simbólica, psicológica ou física. Conforme Tavares dos Santos (2007), a violência é um fenômeno cultural e histórico.

Existem vários instrumentos e tradições sociológicas que nos permitem encontrar ferramentas para a compreensão da violência, já que não há uma teoria geral que dê conta de todas as possíveis reflexões sobre o assunto (WIEVIORKA, 1997). Considerando a perspectiva da sociologia fenomenológica e do interacionismo simbólico, que compartilham a ideia de que os seres humanos agem utilizando os signos que lhes são oferecidos e os significados são consolidados durante o processo interativo ao longo da vida (SCHUTZ, 2012; BLUMER, 1980), uma boa maneira de encontrar a realidade é perguntar sobre ela à pessoa que experiencia os fenômenos (BERGER; LUCKMANN, 2004).

Em uma reflexão sobre a clássica discussão sociológica da oposição entre indivíduo e sociedade, Gilberto Velho ressalta a complexidade de ambos. Recorrendo principalmente ao interacionismo, o autor lembra que os indivíduos transitam entre diversos mundos socioculturais em múltiplos níveis de realidade, agindo em diferentes contextos e situações. Assim como participam da estrutura geral, também vivenciam internamente subjetividades. Para Velho, portanto, a investigação de projetos individuais pode ajudar a entender como os sujeitos organizam e desenvolvem condutas específicas para atingir seus objetivos. "Trata-se de tentar captar os significados e procedimentos dessas ações diante de forças históricas e sociais poderosas das quais nem sempre os agentes individuais são conscientes ou têm uma percepção mais elaborada" (VELHO, 2006, p. 8).

Nesse sentido, a concepção biográfica é importante porque é nela em que está contido todo o processo de reconhecimento do mundo, que começa desde o nascimento. As pesquisas com uso de biografias têm trazido importantes reflexões sobre o engajamento dos indivíduos em ações violentas. Esses métodos que se valem de histórias de vida dão acesso às interpretações dos sujeitos às suas motivações e aos seus roteiros de ação (SANTOS, SUSIN; OLIVEIRA, 2014).

As informações sobre trajetórias de vida contidas em entrevistas, diários, cartas dão acesso ao ponto de vista dos agentes das ações sociais (ROSENTHAL, 2017). Nos anos 1920, são registrados os trabalhos pioneiros nessa área dentro das universidades, sendo o estudo "O lavrador polonês na Europa e nos Estados Unidos", sobre migração, realizado por William Isaac Thomas e Florian Znaniecki, considerado o primeiro. A partir dessa pesquisa, o departamento de sociologia da Universidade de Chicago reconheceu e desenvolveu o método biográfico como uma ferramenta para a apreensão da perspectiva subjetiva e da ação social de integrantes dos mais variados meios e "para a reconstrução de mundos da vida em geral e da aplicação de seus resultados na busca de respostas para questões originadas na práxis social" (ROSENTHAL, 2014, p. 212).

A partir da década de 1970, houve um aumento do interesse pela pesquisa biográfica em diferentes disciplinas e o seu uso ainda se mantém. A releitura dos trabalhos da Escola de Chicago fez 
com que houvesse uma disseminação ainda maior da abordagem biográfica. Atualmente, o método é aplicado em várias áreas da sociologia.

Gabriele Rosenthal (2017) entende que o uso de biografias na pesquisa social ajuda a captar as dinâmicas da relação entre o que está no campo individual e coletivo.

$\mathrm{Na}$ "autoapresentação biográfica" temos acesso não apenas ao processo biográfico de interiorização do mundo social no curso da socialização, mas também à integração das experiências biográficas no estoque de conhecimento e com isso à constituição de esquemas de experiência que servem à orientação atual e futura dentro do mundo social. Essa integração, que constitui as experiências como dotadas de sentido e dá origem à visão geral biográfica e às projeções biográficas do indivíduo diretamente vinculadas a ela, não deve ser compreendida como contingente, individual. Também ela é antes de tudo socialmente constituída (ROSENTHAL, 2017, p. 19).

Entre essas técnicas, o estudo da biografia dos autores de crimes tem aparecido em muitos trabalhos como uma forma de obter informações e também para compreender esse fenômeno. Nas últimas décadas, está mais frequente o interesse por narrativas a partir de entrevistas com o próprio sujeito em conflito com a lei (ARFUCH, 2018). Isso sem citar as autobiografias de indivíduos, incluindo os que vivenciaram a criminalidade e períodos na prisão. Entre alguns exemplos estão David Honeywell (2016) e Safak Bozkurt com Andreas Aresti (2018). O estudo detalhado da vida dos sujeitos, suas relações e os significados atribuídos às suas ações representa um elemento fundamental para entender os sentidos por trás dos fenômenos estruturais nas sociedades.

Nesse contexto, coletar e fornecer uma análise crítica e sistemática da produção acadêmica sobre o assunto permite uma visão reflexiva do que foi investigado. Da mesma forma, uma revisão bibliográfica sobre esse assunto auxilia a descrição de algumas de suas diferentes características centrais, tais como suas especificações metodológicas, linhas de pesquisa e os principais resultados.

Este artigo baseia-se nas seguintes questões: O que foi publicado a partir de uma perspectiva biográfica e narrativa sobre criminosos masculinos e femininos na área das ciências sociais nos últimos dez anos? Como os estudos usaram métodos narrativos e compreensivos para entender as experiências, motivações e interpretações de pessoas que cometem crimes ou exercem alguma forma de violência? Em particular, estamos interessados em saber o que foi investigado sobre mulheres e sobre homens e comparar esses resultados.

Na seção seguinte, descrevemos a estratégia metodológica para a revisão da literatura. Em seguida, apresentamos o resultado do levantamento e a análise crítica e comparativa do que foi encontrado sobre o tema.

\section{Metodologia}

Este artigo se enquadra nas chamadas revisões narrativas (DIXON-WOODS et al., 2006; DIXONWOODS et al., 2007; POPAY E MALLINSON, 2013). Foram coletados artigos e livros publicados e disponíveis nas seguintes bases de dados digitais: SCOPUS e Scientific Electronic Library Online 
(SciELO). Essas plataformas foram escolhidas em razão da a) relevância na América Latina; b) relevância mundial e c) reunião de pesquisas publicadas em milhares de outras fontes.

Para a busca nessas bibliotecas eletrônicas, usamos os seguintes termos: (men OR women) AND (violence OR crime OR offender) AND (biography OR trajectory OR pathway). As pesquisas tanto para homens como para mulheres geraram 1234 títulos na plataforma Scopus e 24 títulos na SciELO (1258 resultados no total). Restringimos o número limitando as datas em um período de dez anos (20092019) e também destacamos apenas trabalhos das áreas das ciências humanas. Escolhemos o período dos últimos dez anos para verificar os estudos e informações mais recentes a respeito do tema.

Como critério geral, esses termos foram utilizados em inglês, uma vez que todas as bases tratam uniformemente as informações nessa língua, embora os artigos sejam escritos em outros idiomas. Selecionamos trabalhos em inglês, espanhol e português. Desse modo, a intenção foi reunir um corpus de análise relativamente homogêneo, já que os trabalhos passaram por avaliação de pares para serem publicados e assim constarem nas bases de dados selecionadas.

Os critérios de inclusão foram: a) artigos indexados que tenham o objetivo de compreender o crime e / ou violência a partir de uma perspectiva biográfica ou narrativa; b) artigos sobre pesquisas empíricas cuja metodologia seja qualitativa com abordagem biográfica ou enfatize trajetórias individuais de homens ou mulheres que tenham praticado crimes.

Critérios de exclusão: a) itens que não abordam crime e / ou principal não tenham como fundamental objetivo a violência, b) revisões bibliográficas, c) artigos com metodologias quantitativas; d) pesquisas não empíricas (comentários, discussões teóricas, etc.).

O processo começou com uma concepção ampla da narrativa e biografia: como o conceito específico envolve algum tipo de coleta de dados técnica (narrativa ou entrevistas biográficas), aqui foram incluídos itens com metodologias com uso de entrevistas semi-estruturadas e abordagem etnográfica. O critério central na seleção dos artigos foi a concentração na coleta e análise de dados sobre aspectos biográficos ou na trajetória de suas vidas. Nenhum cut-out ou filtro geográfico foi aplicado. Após a eliminação de artigos duplicados e para aplicar critérios de inclusão e exclusão, foi formado um corpus final de 56 documentos.

Separamos a pesquisa e a análise sistemática dos trabalhos por gênero. Portanto, em um primeiro momento, buscamos artigos sobre mulheres e depois sobre homens. O formato e conteúdo desses documentos foram verificados mais uma vez de forma separada e, ao final, procuramos elementos de similaridade e de contraste. Observamos prioritariamente o corpus das pesquisas, os objetivos, os métodos utilizados, locais onde ocorreram e resultados.

\section{Estudos com trajetórias de mulheres autoras e seus resultados}

A partir da seleção de pesquisas que utilizam a biografia de mulheres para entender os fenômenos da criminalidade e da violência praticados por elas, foi possível identificar trabalhos com foco no gênero feminino e outros que consideraram e compararam ambos gêneros. A maioria priorizou o estudo de mulheres adultas, mas há alguns específicos sobre jovens infratoras (SHEPHERD et al.; 2018; SENTO-SÉ; COELHO, 2014). Os estudos foram desenvolvidos nos Estados Unidos (SMITH, 
2017; JONES et al., 2018; MCCONAGHY; LEVY, 2016; KENNEDY; MENNICKE, 2018; CARR; HANKS, 2013), Portugal (MATOS, 2018; FROIS, 2017), Iran (MAGHSOUDI et al., 2018), Bélgica (NUYTIENS; CHRISTIAENS, 2016), Austrália (SHEPHERD et al., 2018), Argentina (SALISBURY et al., 2018), Brasil (CORAZZA, 2016; BARCINSKI et al., 2013; SENTO-SÉ; COELHO, 2014), ISRAEL (GUETA; CHEN, 2016) França (HARRATI et al., 2018), Alemanha (KÖTTIG, 2016), Camboja (JEFFRIES; CHUENURAH, 2018), México (MEJÍA-HERNÁNDEZ; WEISS, 2011) e Espanha (PADOVANI, 2016; CALVO, 2017).

Como vimos, o uso de biografias é útil para diferentes temas e enfoques. Os objetivos dessas pesquisas giraram em torno de identificação de motivação para o crime (NUYTIENS; CHRISTIAENS, 2016), explicação para o comportamento desviante (HARRATI et al., 2018), estratégias para lidar com a vida após a prisão (PADOVANI, 2016), demandas de mulheres presas (KENNEDY; MENNICKE, 2018) e uso de drogas (SMITH; 2017).

Entre os métodos para identificar essas trajetórias, o preferido nesse apanhado de pesquisas analisadas foi a entrevista semi-estruturada. Porém, muitos trabalhalhos usaram entrevistas abertas, grupos focais e combinaram as técnicas qualitativas com coleta quantitativa e observação, além de etnografia, por exemplo.

A abordagem feminista como um guia para o desenvolvimento da pesquisa e análise dos dados é recorrente na amostra analisada (CARR; HANKS, 2013; JEFFRIES; CHUENURAH, 2018; SALISBURY ET AL., 2018; MATOS, 2018; SMITH, 2017). Conforme Nicole Carr e Roma Hanks (2013), o feminist pathway approach colabora para o entendimento do envolvimento de mulheres no crime, observando a construção social do gênero como um processo que incluiu mais de um evento ao longo da vida (CARR; HANKS; 2013; p. 433- 434). Salisbury et al. (2018), que também usou essa perspectiva em uma pesquisa sobre a dinâmica em que ocorre a violência e os possíveis fatores de risco para o encarceramento de mulheres na Argentina, identificou que os autores afinados com a abordagem assumem a existência de diferentes realidades biológicas, psicológicas e sociais que são exclusivas da experiência feminina. De acordo com essa abordagem, o desenvolvimento criminoso e a reincidência das mulheres são baseados em fatores: não vistos tipicamente em homens; tipicamente vistos com homens, mas em frequência ainda maior com mulheres; ou vistos em frequência relativamente igual mas com distintos efeitos pessoais e sociais para as mulheres. AS linhas feministas procuram explicar, em geral, como as mulheres acabam em um ciclo de vitimização que leva ao comportamento ofensivo (SALISBURY ET AL.; 2018; p. 130). Nesse sentido, as biografias contribuem para o entendimento das experiências de gênero.

Outras teorias também fundamentaram a escolha do método biográfico, como a Grounded Theory e a Fenomenologia. No artigo intitulado "Patriarchy as a contextual and gendered pathway to crime: A qualitative study of iranian women offenders", publicado em 2018, os autores Maghsoudi, Anaraki, e Boostani usaram a Grounded Theory para analisar a história de 23 mulheres que haviam cometido crimes no Iran. Essa teoria foi escolhida com o intuito de entender o tema do ponto de vista das participantes e não dos pesquisadores.

Os métodos e abordagens utilizadas por esses pesquisadores permitiram chegar a resultados que identificam fatores para o engajamento de mulheres na criminalidade, experiências comuns presentes nas trajetórias das pesquisadas e efeitos. 
Grande parte das pesquisas analisadas apontam a presença de vitimização na trajetória das mulheres pesquisadas (KENNEDY; MENNICKE, 2018; GUETA; CHEN, 2016; BECERRA; SERRA, 2017; KÖTTIG, 2016; NUYTIENS; CHRISTIAENS, 2016; SHEPHERD et al.; 2018; CARR; HANKS, 2013; JEFFRIES; CHUENURAH, 2018; SALISBURY, et al., 2018; SMITH, 2017). Esses trabalhos mostram como as entrevistadas citaram com frequência a ocorrência de violência contra elas perpetrada principalmente pelos pais, companheiros ou pessoas próximas.

Na pesquisa de Luz Adriana Aristizábal Becerra e Jenny Cubells Serra (2017), 92\% das 94 mulheres entrevistadas presas e ex-presas descreveram terem sido vítimas de violência física. A motivação para a criminalidade seria a tentativa de escapar das relações abusivas, por conta do sistema patriarcal.

Michaela Köttig (2016) demonstra, a partir da biografia de uma jovem que acabou se envolvendo com grupos de extrema direita na Alemanha, que o comportamento violento no presente está intimamente vinculado a causas históricas. A personagem que ilustra seu artigo foi espancada e violentada pelo pai, perdeu a mãe ainda criança e sofreu forte pressão psicológica ao longo de sua trajetória. Mais tarde, começou a desenvolver comportamento agressivo. A autora explica que nenhum fenômeno está isolado das demais experiências vividas. Por isso, o significado biográfico é único e não generalizado. A violência, neste caso específico, pode estar ligada a tentativas de reparação e autoconhecimento, mas isso não quer dizer que o mesmo processo ocorreria em outras trajetórias, da mesma forma.

Esses processos complexos podem ser reconstruídos se a sequência no caso individual for preservada e não destruída. Nenhum fenômeno está sozinho e separado de todas as outras experiências e, portanto, seu significado não pode ser descoberto separadamente da gênese completa. O significado de eventos biográficos ou fases de desenvolvimento não pode, portanto, ser generalizado independentemente de sua gênese, por exemplo, assumindo que o comportamento violento sempre tem conotações específicas de gênero ou é sempre destrutivo. Em vez disso, um caso como o de Jacky mostra que a violência pode estar ligada a tentativas de reparação e autoconhecimento. No processo de desenvolvimento, padrões de comportamento são formados de acordo com estruturas regulares (tradução livre, KÖTTIG, 2016, p. 20-22).

Após entrevistar sete mulheres e 13 homens presos, Megan McConaghy e Marissa P. Levy (2016) também observaram a influência da violência na trajetória. As autoras concluíram que a vitimização em ambos sexos é um fator que colabora para o comportamento criminal futuro. Elas identificaram ainda questões que merecem ser melhor investigadas como diferentes razões para abandono entre homens e mulheres, a influência de parceiros criminosos e o impacto do encarceramento precoce versus tardio de homens e mulheres.

Um estudo realizado no sistema penitenciário argentino (SALISBURY et al., 2018) indicou que aquelas que relataram abuso grave eram mais propensas a serem as que cometeram crimes mais sérios contra pessoas (por exemplo, assalto / agressão, assassinato, sequestro, exploração sexual, escravidão reprodutiva ou remoção de órgãos) em vez de crimes relacionados a ganho financeiro. Além de maus-tratos, a falta de suporte social e disruptura familiar são fatores que aparecem relacionados à criminalidade (MAGHSOUDI et al., 2018). As entrevistas semiestruturadas realizadas 
por Shepherd et al. (2018) com adolescentes e jovens adultos de ambos sexos em custódia na Austrália mostraram isso. Entre as questões estavam os temas família, relacionamentos, uso de drogas, dificuldades psicológicas, trabalho, educação e experiências com crime, violências e justiça. Além disso, foram feitos questionários sobre saúde mental dos entrevistados. O estudo mostra que a precoce disruptura familiar contribui para autoria de atos violentos e outros eventos negativos. Muitos entrevistados foram diagnosticados com desordens psicológicas.

Em um trabalho sobre a infância e vida adulta com 41 mulheres presas na Bélgica, Nuytiens e Christiaens (2016) encontraram uma relação entre vulnerabilidade (que pode ser econômica, social, ou afetiva) com o ingresso em ações criminosas e que consequentemente levam à prisão. Eventos ocorridos na vida adulta influenciam mais no crime do que os que registrados na infância delas. Muitas entrevistadas disseram que foram abusadas ou manipuladas por companheiros para cometer crimes. As biografias mostram uma relação desigual de poder entre as mulheres e seus companheiros e isso resulta em dependência e isolamento (NUYTIENS; CHRISTIAENS, 2016).

O trabalho com jovens mulheres na prisão realizado por Raquel Matos (2018) evidenciou que aqueles que se conformam mais aos papéis tradicionais de gênero parecem ser mais vulneráveis a crimes relacionados à sua condição feminina. Mostrou também que o controle social no contexto familiar pode levar as meninas a formas de delinquência tradicionalmente associadas aos meninos. Matos identificou ainda que as perspectivas de profissionais que atuam no sistema carcerário podem ser altamente moldadas por estereótipos de gênero.

Questionando o argumento de alguns trabalhos que identificam a mulher como não agente e, muitas vezes, influenciada por homens, Mariana Barcinski, Carine Capra-Ramos, João L. A. Weber e Tamires Dartora (2013) entrevistaram 20 presas por tráfico em 2011 e 2012. A partir da análise aprofundada das entrevistas, os autores notaram a resiliência das pesquisadas após muitos episódios de sofrimento e trauma em suas trajetórias. Em geral, as identidades dessas pessoas eram muito fortes e elas desempenhavam papéis de protagonismo em várias relações, o que contraria as pesquisas que colocam a mulher em condição apenas de vítima e não de agente de suas escolhas.

Os relacionamentos amorosos, no entanto, foram usados como estratégia para resistir aos traumas e não só como relações violentas. Em pesquisa sobre a rede de relacionamento de mulheres presas, Estibaliz de Miguel Calvo (2017) observou, a partir de 49 entrevistas semiestruturadas e observação participante, que a experiência amorosa não só torna possível superar as dificuldades do aprisionamento, mas altera as lógicas penitenciárias de estranheza e distância e ainda permite reformular a própria identidade.

\section{Produção acadêmica sobre homens autores}

Os estudos que se concentram na análise da biografia dos homens foram desenvolvidos nos Estados Unidos (KRAS E BLASKO, 2016; PANDYA, 2009; RICH; GREY, 2005), Colombia (BAIRD, 2018), Reino Unido (GADD, 2000; ELLIS, 2016; ELLIS; WINLOW; HALL, 2017; HAMMERSLEY et al., 2016; HOLLIGAN; DEUCHAR, 2014; MARTSOLF; DRAUCKER, 2008), Alemania (MOERTL; BUCHHOLZ; LAMOTT, 2010), Nueva Zelanda (LAMBIE; JOHNSTON, 2015; ODGERS et al., 2008), 
Suécia (CARLSSON, 2013; JANSSON, 2018), Chade (DEBOS, 2011), Sudáfrica (DEKEL; ABRAHAMS; ANDIPATIN, 2018), Brasil (GONÇALVES, 2017), Israel (GUETA; CHEN, 2016), Austrália (HONORATO; CALTABIANO; CLOUGH, 2016; LINDSAY, 2012) e Noruega (TJØNNDAL, 2016).

A literatura relacionada a esse tópico não vem de um único campo. Entre as disciplinas estão as Ciências Sociais, em especial a Sociologia (JANSSON, 2017; LINDSAY, 2012; WILKINSON et al., 2009; TJØNNDAL, 2016), a Criminologia (ELLIS, 2016; GUETA; CHEN, 2016), a Criminologia Cultural (CARLSSON, 2013; HOLLIGAN E DEUCHAR, 2014), a Psicologia e os estudos de comportamento (ODGERS et al., 2008; CALTABIANO; CLOUGH, 2016), a Psicanálise (GADD, 2000) e as Ciências Médicas (ELLIS et al., 2017; MARTSOLFT; DRAUCKER, 2008), entre outras.

Como resultado da heterogeneidade de abordagens disciplinares, a literatura sobre homens, crime e biografias diferem tanto em relação às suas metodologias, como nas teorias usadas ou propostas nas investigações. Para considerar especificamente metodologias, encontramos um quadro geral de interesse no uso de dados biográficos e dados biográficos particularmente narrados a partir da perspectiva dos próprios atores. Considerando o quadro metodológico global dos artigos como a investigação a partir de uma abordagem qualitativa, foi encontrada uma variedade de estratégias empregadas: entrevistas narrativas ou entrevistas não-estruturadas, uso de fontes documentais; e o uso mais genérico da perspectiva etnográfica. Da mesma forma, foram localizadas investigações que utilizam a teoria fundamentada.

Embora a maior parte da bibliografia esteja diretamente ligada à abordagem biográfica - e particularmente à narrativa - este método é interpretado a partir de diferentes lentes disciplinares e analíticas. Por exemplo, Honorato et al. (2016) utilizam a técnica de entrevistas em profundidade para investigar fatores de risco para a violência perpetrada por homens.

Além disso, um aspecto metodológico particular desses artigos é que parte deles realizou a coleta de dados em contextos de confinamento. Essa particularidade dos estudos com dados primários sobre violência e criminalidade confere certas características a esses estudos, dentre os quais estão: a) dificuldade ou incapacidade de registrar as entrevistas; b) os obstáculos institucionais para realizar a investigação; c) o desafio de conquistar a confiança dos entrevistados diante do contexto hostil e, ainda, d) as possíveis formas de violência simbólica exercidas sobre os detentos. Estudos como os de Kras e Blasko (2016), Hadden et al. (2016), Honorato, Caltabiano e Clough (2016), Brown (2006) e Gueta e Chen (2016), entre outros, realizaram seus respectivos trabalhos de campo dentro de prisões e identificaram essas barreiras.

No que diz respeito à dimensão teórica, o fato de os artigos virem de diferentes perspectivas disciplinares explica a variedade de orientações analíticas. Nesse sentido, o estudo de biografias de homens com histórico de violência é pensado com base na abordagem de fatores de risco (HONORATO; CALTABIANO; CLOUGH, 2016), a construção de identidades (BAIRD, 2017), a performatividade da masculinidade (GUETA; CHEN, 2016; LINDSAY, 2011) e os impactos psíquicos e emocionais de experiências traumáticas (DEKEL; ABRAHAMS; ANDIPATI, 2018), entre outras. Essa polissemia do termo "biografia" explica a variedade analítica para entender a ligação entre a vida dos sujeitos e suas ações criminosas.

Dentro das teorias utilizadas, chama a atenção que, embora o contexto masculino seja considerado em grande parte dos estudos, ele é reduzido principalmente ao ambiente comunitário e 
familiar. Isto é, as redes de pertencimento aos homens. Ao contrário, os contextos mais gerais e estruturais, como o político-econômico, são analisados apenas em alguns estudos, como Ellis (2016):

Eu quero que o leitor, e os homens que participaram [deste estudo], reconheçam e compreendam que as brutalidades ocasionais perpetradas por e nas minorias do grupo de homens gravemente marginalizados ocorrem no contexto da insegurança e das comunidades desindustrializadas do capitalismo avançado (tradução livre, ELLIS, 2016, p. 14).

Ao considerar o conjunto de bibliografia, quatro grandes grupos podem ser classificados, de acordo com suas perspectivas teóricas e os tópicos de análise: literatura sobre trauma, abuso infantil, gênero / masculinidade e, finalmente, programas de intervenção. Em primeiro lugar, uma parte significativa da bibliografia reunida enfatiza a análise de traumas nas biografias. A recorrência deste conceito pode ser traçada em grande parte dos estudos coletados (HONORATO; CALTABIANO; CLOUGH, 2016; RICH; GREY, 2005; ELLIS; WINLOW; HALL, 2017, DEKEL; ABRAHAMS; ANDIPATIN, 2018; HAMMERSLY et al., 2016). No entanto, o uso e o significado desse conceito são variados. $\mathrm{Na}$ literatura pesquisada, dois tipos principais de definições podem ser apontados. Um se refere a uma definição genérica, na qual o trauma é estabelecido como condição de ações futuras. Por exemplo, "o trauma pode incluir doença física ou lesão, dano, incapacidade, tortura, prisão ou perseguição, dissolução do relacionamento, perda de trabalho, migração / realocação, violência e abuso sexual" (tradução livre, HONORATO; CALTABIANO; CLOUGH, 2016, p. 1).

Uma perspectiva diferente está relacionada a uma concepção simbólica e construtiva, em que o trauma é destacado como um produto de relações e situações que são interpretadas pelos homens. Nesta linha, em um estudo etnográfico no norte da Inglaterra, Ellis, Winlow e Hall (2017) sugerem que, embora possa haver um nexo causal entre experiências traumáticas durante a infância e a persistência de práticas violentas na vida adulta, é necessário entender as especificidades culturais e sociais de cada população.

A experiência traumática, por si só, não é suficiente para levar o indivíduo à violência. $O$ indivíduo deve ser submetido a uma socialização de gênero que enfatize a tenacidade, o estoicismo e a fisicalidade. $O$ indivíduo traumatizado deve ser encorajado a valorizar a violência. (...) Os homens com quem falamos têm ambos os elementos cruciais: eventos biográficos traumáticos no passado que raramente são conscientemente reconhecidos e não podem ser transcendidos emocionalmente, $\mathrm{e}$ uma socialização inculturada que enfatiza a necessidade de enfrentar violentamente qualquer desafio (tradução livre, ELLIS; WINLOW; HALL, 2017, p. 14).

Em segundo lugar, a análise do abuso infantil na bibliografia é recorrente. Este fenômeno aparece principalmente como um fator condicionante nas ações subsequentes criminosas ou violentas dos homens. Isso é principalmente trabalhado em artigos de Psicologia, Criminologia e Ciências Médicas (HAMMERSLEY et al., 2016; HONORATO; CALTABIANO; CLOUGH, 2016; LAMBIE; JOHNSTON, 2011; MARTSOLF; DRAUCKER, 2008; entre outros). Como Lambie e Johnston (2011) referem, essas investigações são guiadas pela hipótese do abusador sexual abusado sexualmente (sexually abused-sexual abuser hypothesis). 
Um terceiro grupo de literatura aborda as biografias em relação a homens de uma perspectiva de identidade e gênero (BAIRD, 2017; CARLSSON, 2013; DEBOS, 2011; GUETA; CHEN, 2012; JANSSON, 2018; LINDSAY, 2012; MOERTL; BUCHHOLZ; LAMOTT, 2010). Esses estudos mostram uma interpretação mais construtivista da abordagem narrativa / biográfica, investigando especificamente os processos de socialização da masculinidade, construção identitária e atribuição de significados.

Nessa perspectiva, Debos (2011) analisa as formas de violência armada cotidiana no Chade a partir da hipótese de que a violência armada é um modo cotidiano de expressar rebelião, como uma forma de ocupação prática, o métier. Em um estudo de biografias de gangues masculinas em Medellin, Baird (2017) observa que muitos dos jovens se juntam a gangues para simular e reproduzir identidades masculinas locais "bem-sucedidas". Como o autor argumenta, unir-se a uma gangue "não deve ser entendido em termos de comportamento juvenil desviante, mas como um produto de uma lógica prática ao se juntar a uma gangue como um espaço de treinamento de identidade para jovens que crescem em condições de exclusão estrutural" (tradução livre, BAIRD, 2017, p. 1). Por sua vez, Carlsson (2013), Tjønndal (2016) e Lindsay (2012) apontam que, para compreender as ações ou práticas de violência e crime, é necessário investigar as normas específicas de cada contexto cultural.

Dentro dessa grande estrutura de estudos de gênero, Lindsay (2012) levanta duas críticas aos estudos tradicionais de gênero, biografia e violência que dificultam uma visão linear desse campo temático. Em primeiro lugar, em seu estudo sobre masculinidade, consumo de álcool e violência, a autora enfatiza que é necessário repensar a premissa de que a identidade de gênero é vivenciada de forma positiva pelos atores: ou seja, que as práticas associadas à masculinidade não são sempre positivamente percebidas. Em segundo lugar, sugere que é conveniente desenvolver linhas de pesquisa que promovam uma noção dinâmica de gênero, na qual seus processos de negociação, suas heterogeneidades e as transformações que ocorrem sejam levadas em conta.

Um grupo final de estudos aborda programas ou estratégias de intervenção socioeducativa. Tanto Pandya (2009) quanto Gonçalves (2017) e Kras e Klasko (2016) analisam as mudanças emocionais e atitudinais dos homens em aparelhos grupais após a perpetração de crimes (crime sexual e prisão por outros crimes). Em todos os estudos, as respostas dos homens foram modeladas contra essas intervenções e, em todos os casos, destaca-se a dificuldade de delimitar as atitudes e os modos desses homens após a participação nessas intervenções.

\section{De vítima à agressor: o espaço para a vitimização na sociedade}

Em razão de a ideia de vitimização perpassar, em larga medida, a bibliografia sobre "autoria de violência", se faz necessária uma breve reflexão sobre esta categoria. Destacamos aqui duas abordagens para o tema. O primeiro é a consideração da vítima na dinâmica de violência em que ela se apresenta em uma situação desfavorável de poder em relação ao agressor (TAVARES DOS SANTOS, 2007) e que merece lugar na sociedade como sujeito a ser escutado.

A autora Cynthia Sarti (2014) analisa que, diferente de outros sentimentos que são expressos e inteligíveis por meio da linguagem, o sofrimento decorrente da violência é somado ao sofrimento por não haver maneiras de expressão da dor. Normalmente, por causa da negação ou humilhação, 
ocorre um silêncio sobre o assunto. Segundo a pesquisadora, é inesgotável, incompleta e inacabada a tarefa de escutar e pensar a dor do outro em circunstâncias de violência.

Para a construção social da vítima, é importante observar o agressor e o contexto da violência, que permitem entender a lógica a partir da qual ela se manifesta e é qualificada. Nesse sentido, ser vítima não corresponde a um espaço imutável em razão do poder circular (SARTI, 2011).

Pensando nisso, destacamos outra abordagem para o tema que é a mudança de papéis do sujeito que em algumas situações é vítima e em outras agressor. Os dados extraídos das pesquisas analisadas neste artigo mostram que há um grande número de pessoas que cometeram violência e que tinham em suas biografias episódios de vitimização anterior. São pessoas que sofreram uma ou mais de uma agressão física e psicológica e que também foram autoras. Esse resultado mostra a pertinência de se problematizar essa questão e ver o tema de forma mais dinâmica e menos estática.

\section{Considerações finais}

Em relação às perspectivas metodológicas e teóricas, tanto a literatura sobre mulheres quanto sobre homens apresenta similaridades. Em ambos os grupos, as técnicas de coleta de dados utilizadas são variadas, mesmo dentro da estrutura geral dos estudos biográficos.

Da mesma forma, em ambos os grupos de bibliografia, foi encontrada a recorrência de teorias de gênero e identidade para o estudo desses tópicos. Tanto do feminist pathway approach (CARR E HANKS, 2013), a partir de estudos sobre masculinidades (LINDSAY, 2012; BAIRD, 2017) e criminologia cultural (CARLSSON, 2013) a dimensão de gênero aparece como uma variável explicativa das ações e significados em torno da violência.

Outro aspecto comum é que as trajetórias biográficas de mulheres e homens parecem marcadas por experiências de vitimização. Na literatura analisada, a vida das pessoas que cometeram crimes ou ações violentas é imbricada com eventos significativos de violência. No entanto, há uma discordância nos autores sobre o que significam as experiências de vitimização nessas populações: enquanto alguns enfatizam que esses eventos da juventude condicionaram essas pessoas em suas práticas subsequentes (HONORATO, CALTABIANO E CLOUGH, 2016), outros apontam que a reprodução da violência e do crime só ocorre nos casos em que há condições de possibilidade social e cultural (ELLIS, WINLOW E HALL, 2017).

No contexto dessas hipóteses, existe uma variedade de estudos que pesam de maneira diferente a ligação entre a vitimização na própria vida e o exercício da violência sobre os outros. Uma diferença notável é que nos estudos sobre o sexo masculino, pesquisas focadas em medidas e dispositivos socioeducativos (dispositivos para homens que praticaram violência doméstica ou violência sexual) foram registradas, enquanto nenhum estudo similar foi registrado para a população feminina. No caso dos trabalhos com homens, a maioria concentrou a amostra em jovens. Já as pesquisas com mulheres, a maior parte tem entrevistas com adultas, o que demonstra a diferença de objetivos e interesses. 


\section{REFERÊNCIAS}

ARFUCH, Leonor. La vida narrada. Villa María: Eduvim, 2018. https://doi.org/10.24215/18517811e115 BAIRD, Adam. Becoming the 'Baddest': Masculine Trajectories of Gang Violence in Medellín. Journal of Latin American Studies, [s. l.], n. 50, v. 1, p. 183-210, 2018. https://doi.org/10.1017/s0022216x17000761 BARCINSKI, Mariana; CAPRA-RAMOS, Carine; WEBER, João; DARTORA, Tamires. O marianismo e vitimização de mulheres encarceradas: formas alternativas de exercício do poder feminino. Exæquo, [s.l.], n. 28, p. 87-100, 2013.

BECERRA, Luz Adriana Aristizábal; SERRA, Jenny Cubells. Female Delinquency and Withdrawal: Explanatory Factors. Universitas Psychologica, Bogotá, n. 16, v. 4, 2017.

BERGER, Peter; LUCKMANN, Thomas. A construção social da realidade: tratado de sociologia do conhecimento. Tradução: Floriano de Souza Fernandes. Petrópolis: Vozes, 2004.

BLUMER, Herbert. A natureza do interaccionismo simbólico. In: Mortensen, C. D. Teoria da comunicação: textos básicos. São Paulo: Mosaico, 1980.

BOZKURT, Safak; ARESTI, Aresti. Absent voices: Experiencing Prison Life From Both Sides of the Fence. A Turkish Females Perspective. Journal of Prisoners on Prisons, [s. l.], v. 27, n. 2, p. 17-36, 2018.

CALVO, Estabaliz de Miguel. Explorando la agencia de las mujeres encarceladas a través de sus experiencias amorosas. Papers 2017, [s. l.], v. 102, n. 2, p. 311-335, 2017. https://doi.org/10.5565/ rev/papers.2340

CARLSSON, Christoffer. Masculinities, Persistence, and Desistance. Criminology, [s. l.], n. 51, v. 3 , p. 661-693, 2013. https://doi.org/10.1111/1745-9125.12016

CARR, Nicole; HANKS, Roma. Everything I've Done I've Done for MeN : One Woman's Deployment of Femininities and Her Pathway to Crime. Sociological Spectrum, [s. l.], v. 33, n. 5, p. 433-452, 2013. https://doi.org/10.1080/02732173.2013.818507

CORAZZA PADOVANI, Natalia. Plotting Prisons, Flows and Affections: Brazilian Female Prisoners between the Transnational Drug Trade and Sex Markets in Barcelona. Criminology \& Criminal Justice, [s. l.], v. 16, n. 3, p. 366-385, 2016. https://doi.org/10.1177/1748895816646611

DEBOS, Marielle. Living by the gun in Chad: Armed violence as a practical occupation. Journal of Modern African Studies, [s. l.],v. 3, n. 49, p. 409-428, 2011. https://doi.org/10.1017/s0022278x11000267

DEKEL, Bianca; ABRAHAMS, Naeemah; ANDIPATIN, Michelle. Exploring adverse parent-child relationships from the perspective of convicted child murderers: A South African qualitative study. PLoS ONE, [s. l.], v. 5, n. 13, 2018. https://doi.org/10.1371/journal.pone.0196772

DIXON-WOODS, Mary; BOOTH, Andrew; SUTTON, Alex. Synthesizing qualitative research: a review of published reports. Qualitative Research, v. 7, n. 3, p. 375-422, 2007. https://doi. org/10.1177/1468794107078517

DIXON-WOODS, Mary; CAVERS, Debbie; AGARWAL, Shona; ANNANDALE, Ellen; ARTHUR, Antony; HARVEY, Janet; HSU, Ron; KATBAMMA, Savita; OLSON, Richard; SMITH, Lucy, RILEY, Richard; SUTTON, Alex. Conducting a critical interpretative synthesis of the literature on access to healthcare by vulnerable groups. BMC Medical Research Methodology, [s. l.],v. 6, n. 35, 2006. https:// doi.org/10.1186/1471-2288-6-35

ELLIS, Anthony. Men, masculinities and violence: an ethnographic study. Devon: Routledge, 2016. 
ELLIS, Anthony; WINLOW, Simone; HALL, Steve. 'Throughout my life I've had people walk all over me': Trauma in the lives of violent men. Sociological Review, v. 4, n. 65, p. 699-712, 2017. https:// doi.org/10.1177/0038026117695486

FROIS, Cataria. Female imprisonment. An ethnography of everyday life in confinement. Lisboa: Cham Springer International Publishing, 2017.

GADD, David. Masculinities, Violence and Defended Psychosocial Subjects. Theoretical Criminology, [s. l.], v. 4, n. 4, p. 429-449, 2000. https://doi.org/10.1177/1362480600004004002

GONÇALVES, Rosângela. Entre o mundão e a casa: A passagem pelo centro de atendimento socioeducativo ao adolescente (fundação casa) e a aproximação aos códigos e procederes do sistema prisional. Dilemas, [s. l.], v. 3, n. 10, p. 449-478, 2017.

GROSSI PORTO, Maria Stela; AZEVEDO, Rodrigo Ghiringhelli de. Apresentação dossiê Violência e Sociedade. Civitas, Porto Alegre, v. 13, n. 1, p. 5-8, 2013.

GUETA, Keren; CHEN, Gila. Men and Women Inmates' Accounts of Their Pathways to Crime: A Gender Analysis. Deviant Behavior, [s. l.], v. 12, n. 37, p. 1459-1472, 2016. https://doi.org/10.1080/ 01639625.2016 .1189753

HADDEN, Jennifer; THOMAS, Susan; JELLICOE-JONES, Lorna; MARSH, Zoe. An exploration of staff and prisoner experiences of a newly commissioned personality disorder service within a category B male establishment. Journal of Forensic Practice, [s. l.], v. 3, n. 18, p. 216-228, 2016. https://doi. org/10.1108/jfp-08-2015-0044

HAMMERSLEY, Richard; DALGARNO, Phil; MCCOLLUM, Sean; REID, Marie; STRIKE, Yvonne; SMITH, A.; WALLACE, Jason; SMART, Audry; JACK, Moira; THOMPSON, Alan; LIDDELL, David. Trauma in the childhood stories of people who have injected drugs. Addiction Research and Theory, v. 2, n. 24, p. 135-151, 2016. https://doi.org/10.3109/16066359.2015.1093120

HARRATI, Sonia; COULANGES, Mathilde; DERIVOIS, Daniel; VASSORI, David. Qualitative study on the traumatic experiences of female sex offenders. Journal of Loss and Trauma, [s. l.], v. 23, n. 4, p. 271-286, 2018. https://doi.org/10.1080/15325024.2018.1436869

HOLLIGAN, Chriss; DEUCHAR, Ross. What does it mean to be a man? Psychosocial undercurrents in the voices of incarcerated (violent) Scottish teenage offenders. Criminology and Criminal Justice, [s. l.], v. 3, n. 15, p. 361-377, 2015. https://doi.org/10.1177/1748895814545407

HONEYWELL, David. Returning to Durham prison 30 years on: An ex-prisoners perspective. Prison Service Journal, [s. l.], v. 22, p. 14-20, 2016.

HONORATO, Bronwyn; CALTABIANO, Nerina; CLOUGH, Alan. From trauma to incarceration: exploring the trajectory in a qualitative study in male prison inmates from north Queensland, Australia. Health and Justice, [s. l.], v. 1, n. 4, p. 1-10, 2016. https://doi.org/10.1186/s40352-016-0034-X

JANSSON, Peter. Exploring Pathways Related to Men's Violence: A Qualitative Exploration of the Relationship between Violent Men's Violence and Their Masculinities, Childhood, and Emotions. Deviant Behavior, [s. l.], v. 16, n. 1, p. 1-17, 2018. https://doi.org/10.1080/01639625.2018.1472929

JEFFRIES, Samantha; CHUENURAH, Chontit. Pathways to prison in Cambodia for homicide offending: Exploring women's life history narratives. South East Asia Research, v. 26, n. 2, p. 109-13, 2018. https://doi.org/10.1177/0967828x18769223

JONES, Melissa; SHARP, Susan; WORTHEN, Meredith. Broken Hearts and Battered Lives: Adverse and Abusive Life Histories and Externalized Responses to Anger as Pathways to Illicit Drug Use 
Among Incarcerated Women. Women \& Criminal Justice, [s. l.], v. 28, n. 3, p. 167-188, 2018. https:// doi.org/10.1080/08974454.2017.1372329

KENNEDY, Stephanie; MENNICKE, Annelise. Behind every woman in prison is a man": Incarcerated Women's Perceptions of How We Can Better Help Them in the Context of Interpersonal Victimization. Journal of Progressive Human Services, [s. l.], v. 29, n. 3, p. 206-229, 2018. https://doi.org/10.108 o/10428232.2017.1399034

KÖTTIG, Michaela. The causes of violent actions by young women. Civitas, Porto Alegre, v. 16, n. 1, p. 8-25, jan./mar. 2016.

KRAS, Kimberly; BLASKO, Brandy. Pathways to Desistance among Men Convicted of Sexual Offenses. International Journal of Offender Therapy and Comparative Criminology, [s. l.], v. 15, n. 60, p. 17381755, 2016. https://doi.org/10.1177/0306624x16668178

LAMBIE, Ian; Johnston, Emma. I Couldn't Do It to a Kid Knowing What It Did to Me. International Journal of Offender Therapy and Comparative Criminology, [s. l.], v. 8, n. 60, v. 8, p. 897-918, 2011. https://doi.org/10.1177/0306624x14567664

LINDSAY, Joanne. The gendered trouble with alcohol: Young people managing alcohol related violence. International Journal of Drug Policy, [s.l.], v. 3, n. 23, p. 236-241, 2012. https://doi.org/10.1016/j. drugpo.2011.12.002

MAGHSOUDI, Aliasghar; ANARAKI, Nahid Rahimipour; BOOSTANI, Dariush. Patriarchy as a contextual and gendered pathway to crime: a qualitative study of Iranian women offenders. Quality \& Quantity: International Journal of Methodology, [s. l.], v. 52, n. 1, p. 355-370, 2018. https://doi. org/10.1007/s11135-017-0470-2

MARTSOLF, Donna; DRAUCKER, Claire. The legacy of childhood sexual abuse and family adversity. Journal of Nursing Scholarship, v. 4, n. 40, p. 333-340, 2008. https://doi.org/10.1111/j. 1547-5069.2008.00247.x

MATOS, Raquel. Gender and Crime in the Life Pathways of Young Women Offenders: Contrasting the Narratives of Girls and Professionals. In: GOMES, Sílvia; DUARTE, Vera (org). Female Crime and Delinquency in Portugal. Maia: Palgrave Macmillan, 2018, p. 163-182. https://doi.org/10.1007/9783-319-73534-4 9

MCCONAGHY, Megan; LEVY, Marissa P. The Impact of Gender and Early Delinquency on Reoffending: A Life History Perspective. Victims \& Offenders, [s. l.], v. 11, n. 2, p. 251-284, 2016. https://doi.org /10.1080/15564886.2014.976352

MEJIA-HERNANDEZ, Juana María; WEISS, Eduardo. La violencia entre chicas de secundaria. RMIE, [s. l.], v. 16, n. 49, p. 545-570, 2011.

MOERTL, Kathrin; BUCHHOLZ, Michael; LAMOTT, Franziska. Gender constructions of male sex offenders in Germany: Narrative analysis from group psychotherapy. Archives of Sexual Behavior, [s. l.], v. 39, n. 1, p. 203-2012, 2010. https://doi.org/10.1007/s10508-009-9588-1

NUYTIENS, A.; CHRISTIAENS, J. Female pathways to crime and prison: Challenging the (US) gendered pathways perspective. European Journal of Criminology, v. 2, n.13, p. 195-213, 2016. https:// doi.org/10.1177/1477370815608879

ODGERS, Candice; MOFFITT, Terrie; BROADBENT, Jonathan; DICKSON, Nigel; HANCOX, Robert; HARRINGTON, Honalee; POULTON, Richie; SEARS, Malcom; THOMSON, Murray; CASPI, Avshalom. Female and male antisocial trajectories: From childhood origins to adult outcomes. Development and Psychopathology, [s. l.], v. 2, n. 20, p. 673-716, 2008. https://doi.org/10.1017/s0954579408000333 
PANDYA, Varsha. A study of change processes in domestically violent men in group therapy. Journal of Evidence-Based Social Work, [s. l.], v. 2, n. 6, p. 127-146, 2009. https://doi. org/10.1080/15433710802533348

POPPAY, Jennie; MALLINSON, Sara. Qualitative research review and synthesis. In: BOURGEAULT, I.; DINGWALL, R.; VRIES, R. The SAGE Handbook of Qualitative Methods in Health Research. London: SAGE, 2013, p. 289-306. https://doi.org/10.4135/9781446268247

$\mathrm{RICH}$, John; GREY, Courtney. Pathways to recurrent trauma among young Black men: Traumatic stress, substance use, and the "code of the street". American Journal of Public Health, [s. l.], v. 5, n. 95, p. 816-824, 2005. https://doi.org/10.2105/ajph.2004.044560

SALISBURY, Emily; KALANTRY, Sital; BOPPRE, Breanna; BRUNDIGE, Elizabeth; MARTÍNEZ, Silvia. Expanding the Feminist Pathways Perspective beyond the United States: A Profile of Federal Women Prisoners in Argentina. Women \& Criminal Justice, [s. l.], v. 28, n. 2, p. 125-151, 2018. https://doi.or g/10.1080/08974454.2017.1342745

SANTOS, Hermílio; SUSIN, Priscila; OLIVERIA, Patrícia. Narrativas e pesquisa biográfica na sociologia brasileira: Revisão e perspectivas. Civitas, Porto Alegre, v. 14, n. 2, p. 359-382, 2014. https://doi. org/10.15448/1984-7289.2014.2.17152

SANTOS, José Tavares dos. As lutas sociais contra as violências. Política \& Sociedade, Florianópolis, v. 6, n. 11, p. 70-100, 2007.

SANTOS, José Tavares dos. Violências e Conflitualidades. Porto Alegre: Tomo Editorial, 2009. (Série Sociologia das Conflitualidades). https://doi.org/10.5801/ncn.v2111.5775

SARTI, Cynthia. A construção de figuras da violência: a vítima, a testemunha. Horizontes Antropológicos, Porto Alegre, n.42, 2014. https://doi.org/10.1590/s0104-71832014000200004

SARTI, Cynthia. A vítima como figura contemporânea. Caderno CRH, [s. l.], v. 24, n. 61, p. 51-63, jan./abr. 2011. https://doi.org/10.1590/s0103-49792011000100004

SCHUTZ, Alfred; WAGNER, Helmut T. R. (org.). Sobre fenomenologia e relações sociais.. Petrópolis, Rio de Janeiro: Vozes, 2012.

SENTO-SÉ, João; COELHO, María Claudia. Sobre errâncias, imprecisões e ambivalências: Notas sobre as trajetórias de jovens cariocas e sua relação com o mundo do crime. Horizontes Antropológicos, Porto Alegre, v. 20, n. 42, p. 327-357, 2014. https://doi.org/10.1590/s0104-71832014000200013

SHEPHERD, Stephane, NEWTON, Danielle; HARRIES, Cieran; FIX, Rebecca; FULLAM, Rachel. An analysis of high-risk offending pathways for young females in custody. Psychiatry, Psychology and Law, [s. l.], 2018. https://doi.org/10.1080/13218719.2018.1487344

SMITH, Vivian. Substance-Abusing Female Offenders as Victims: Chronological Sequencing of Pathways Into the Criminal Justice System. Victims \& Offenders, [s. l.] v. 12, n. 1, p. 113-137, 2017. https://doi.org/10.1080/15564886.2015.1017131

TJØNNDAL, Anne. N. H. Heavyweights: Narratives of violence and masculinity in ice hockey. Physical Culture and Sport, Studies and Research, [ s. l.], v.1, n. 70, p. 55-68, 2016. https://doi.org/10.1515/ pcssr-2016-0013

WIEVIORKA, Michel. O novo paradigma da violência. Tempo Social, São Paulo, v. 9, n. 1, p. 5-41, 1997. VELHO, Gilberto. Ciências Sociais e biografia individual. Aula Inaugural. 2006. 
Recebido: 30/05/2019

Aceito: 04/12/2019

\section{Biografia do Autor}

\section{Karina Schuh Reif}

Doutora pelo Programa de Pós-Graduação em Ciências Sociais na Pontifícia Universidade Católica do Rio Grande do Sul (Pucrs). Pesquisa vivências de mulheres autoras de violência e com passagem pela prisão com o método Narrativas Biográficas, a partir de uma leitura fenomenologicamente fundamentada, também utilizando aparatos etnográficos. É mestre em Ciências Sociais e graduada em Jornalismo pela Pucrs, em 2006. Atuou em assessorias de imprensa e veículos de comunicação de Porto Alegre, incluindo o jornal Correio do Povo, onde trabalhou por sete anos. Tem experiência em reportagens para internet e para impresso, nas áreas de Geral, Ensino, Economia e Polícia. ORCID: http://orcid.org/o0o0-0001-8589-8777; EMAIL: karinareif@gmail.com.

Instituição: Pontifícia Universidade Católica do Rio Grande do Sul.

Localização: Av. Ipiranga, 6681 -Partenon - Porto Alegre/RS

\section{Martín Hernán Di Marco}

Mestre e doutorando em Ciências Sociais; ORCID http://orcid.org/0000-0002-0568-0581; EMAIL: mh.dimarco@gmail.com.

Instituição: Universidad de Buenos Aires, UBA, Argentina.

Localização: Av. Ipiranga, 6681 -Partenon - Porto Alegre/RS 